\title{
Two new missense mutations in the protein interaction ASH domain of OCRL1 identified in patients with Lowe syndrome
}

\author{
Ana Perdomo-Ramirez ${ }^{1}$, Montserrat Antón-Gamero ${ }^{2}$, Daniela Sakaguchi Rizzo ${ }^{3}$, \\ Amelia Trindade ${ }^{4}$, Elena Ramos-Trujillo ${ }^{1}$, Felix Claverie-Martin ${ }^{1, *}$ \\ ${ }^{1}$ Unidad de Investigación, Hospital Universitario Nuestra Señora de Candelaria, Santa Cruz de Tenerife, Spain; \\ ${ }^{2}$ Nefrología Pediátrica, Hospital Universitario Reina Sofía, Córdoba, Spain; \\ ${ }^{3}$ Medical Center, Sao Carlos, Sao Paulo, Brazil; \\ ${ }^{4}$ Departamento de Medicina, Universidade Federal de Sao Carlos, Sao Paulo, Brazil.
}

SUMMARY The oculocerebrorenal syndrome of Lowe is a rare X-linked disease characterized by congenital cataracts, proximal renal tubulopathy, muscular hypotonia and mental impairment. This disease is caused by mutations in the $O C R L$ gene encoding membrane bound inositol polyphosphate 5-phosphatase OCRL1. Here, we examined the OCRL gene of two Lowe syndrome patients and report two new missense mutations that affect the ASH domain involved in protein-protein interactions. Genomic DNA was extracted from peripheral blood of two non-related patients and their relatives. Exons and flanking intronic regions of $O C R L$ were analyzed by direct sequencing. Several bioinformatics tools were used to assess the pathogenicity of the variants. The threedimensional structure of wild-type and mutant ASH domains was modeled using the online server SWISS-MODEL. Clinical features suggesting the diagnosis of Lowe syndrome were observed in both patients. Genetic analysis revealed two novel missense variants, c.1907T $>$ A (p.V636E) and c.1979A $>$ C (p.H660P) in exon 18 of the OCRL gene confirming the clinical diagnosis in both cases. Variant c.1907T $>$ A (p.V636E) was inherited from the patient's mother, while variant c.1979A $>$ C (p.H660P) seems to have originated de novo. Analysis with bioinformatics tools indicated that both variants are pathogenic. Both amino acid changes affect the structure of the OCRL1 ASH domain. In conclusion, the identification of two novel missense mutations located in the OCRL1 ASH domain may shed more light on the functional importance of this domain. We suggest that p.V636E and p.H660P cause Lowe syndrome by disrupting the interaction of OCRL1 with other proteins or by impairing protein stability.

Keywords $\quad O C R L$, Lowe syndrome, missense mutation, ASH domain

\section{Introduction}

The oculocerebrorenal syndrome of Lowe (OMIM \#309000) is a rare X-linked disorder characterized by congenital cataracts, intellectual impairment, and progressive renal tubular dysfunction $(1,2)$. Other features include muscular hypotonia, severe growth retardation and behavioral problems. Cataracts and muscular hypotonia are evident at birth whereas the proximal tubulopathy is frequently diagnosed later in life. The tubular dysfunction involves low-molecularweight proteinuria, aminoaciduria and hypercalciuria, and usually results in progressive renal failure leading to end-stage renal disease in adulthood $(1,3)$. The disease is caused by mutations in the OCRL gene encoding a membrane bound inositol polyphosphate 5-phosphatase
(OCRL1), which dephosphorylates the 5' position of the inositol ring in membrane phospholipids (4). The $O C R L$ gene is located on chromosome $\mathrm{Xq} 26.1$ and is expressed in nearly all tissues (5). It contains 24 exons including exon $18 \mathrm{a}$, which is alternatively spliced and mostly expressed in brain $(4,6)$. Lowe syndrome affects almost exclusively males, although a few female carriers with the clinical symptoms of the disease have been reported $(7,8)$. Interestingly, some mutations of the OCRL gene cause Dent-2 disease (OMIM \#300555), which presents a similar proximal tubule dysfunction but only mild or no additional clinical defects (9-11). There seems to be a continuous range of symptoms, varying from very severe features of Lowe syndrome to mild forms of Dent-2 disease that are mostly restricted to the kidney (1). In most cases, genotype-phenotype 
correlations have not been established.

OCRL1 is a multi-domain protein that is comprised of an N-terminal pleckstrin homology $(\mathrm{PH})$ domain followed by a 5-phosphatase catalytic domain, an ASPM-SPD-2-Hydin (ASH) domain, and a C-terminal RhoGAP-like domain that is catalytically inactive (12-14). The PH domain, ASH domain and RhoGAPlike domain specify the subcellular localization and function of the enzyme. The OCRL1 protein localizes predominantly at the trans-Golgi network and early endosomes, and is also present at the plasma membrane, endocytic clathrin-coated pits and vesicles, intercellular junctions of polarized cells, and primary cilium (2). This wide subcellular distribution is mediated by its interactions with different proteins, such as clathrin heavy chain, the clathrin adaptor AP2, several Rab and Rho GTPases, and the endocytic proteins APPL1, Ses1 and Ses2. The ASH-RhoGAP module regulates the interactions of OCRL1 with many proteins. In connection with its diverse cellular locations and interactions, OCRL1 is involved in different functions including endocytic trafficking, cell migration and polarity, actin polymerization and ciliogenesis (2). However, its precise role in disease is not fully comprehended.

Lowe syndrome has been described in all ethnicities and its prevalence in the general population has been estimated to be between 1:500,000 and 1:1,000,000 $(1,2,15)$. Approximately $63 \%$ of $O C R L$ mutations identified in patients with Lowe syndrome are nonsense, frameshift, or splice site mutations that lead to premature termination of the OCRL1 protein or mRNA decay, while missense mutations comprise about 33\% (16-18). Most mutations causing Lowe syndrome are located in exons 9-23, and affect the 5-phosphatase, the ASH and the RhoGAP-like domains (2). Missense mutations in the ASH and RhoGAP domains usually abolish interactions with other proteins and affect OCRL1 targeting (19-23).

Here, we report the identification of novel missense variants affecting the ASH domain of OCRL1 in two patients with Lowe syndrome. The potential pathogenic consequences of these two variants were evaluated using bioinformatics tools and protein modeling.

\section{Materials and Methods}

\subsection{Patients and genetic analysis}

The study included two non-related affected male patients who fulfilled the classically recognized criteria of Lowe syndrome diagnosis including mental retardation, bilateral congenital cataract, congenital hypotonia, and tubulopathy. One child originated from Brazil and the other one from Spain. Neither of them had a family history of the disease. Written informed consent for the genetic analysis was obtained from the patient's parents. The Ethics Committee of Hospital Universitario Nuestra Señora de Candelaria (Santa Cruz de Tenerife, Spain) approved the protocols of this study, which was conducted according to the Declaration of Helsinki.

We performed a mutational analysis of the affected patients and their relatives by direct DNA sequencing. Genomic DNA was extracted from peripheral blood samples using the Gen Elute Blood Genomic DNA kit (Sigma-Aldrich, St. Louis, MO, USA) following the manufacturer's instructions. Coding exons and intronic flanking regions of $O C R L$ were amplified by polymerase chain reaction (PCR) using intronic primers previously described (9). PCR products were purified with the NucleoSpin Gel and PCR Clean-up (MachereyNagel, Düren, Germany). Purified products were sent out to Macrogen Spain Inc. (Madrid, Spain) for DNA sequencing. Mutations were confirmed by sequencing additional independent amplification products. Variant position was based on the cDNA sequence (Ensembl $O C R L$ transcript ID: ENST00000371113.9) using the first coding ATG of exon 1 as initiation codon. We examined several databases, including Human Gene Mutation database (HGMD, http://www.hgmd. cf.ac.uk/ac/index.php), ClinVar (https://www.ncbi. nlm.nih.gov/clinvar), 1000 Genomes Project (http:// www.1000genomes.org/) and gnomAD database (https:// gnomad.broadinstitute.org/), to verify that the variants detected were not common polymorphism and to confirm that they were novel.

\subsection{Bioinformatics analysis}

The potential functional effect of amino acid substitutions generated by the variants on the OCRL1 protein was assessed using several bioinformatics tools including SIFT (http://sift.bii.a-star.edu.sg/www/SIFT seq_submit2.html) (24), PolyPhen-2 (http://genetics. bwh.harvard.edu/pph2/) (25), CADD (https://cadd. gs.washington.edu/) (26), MutPred2 (http://mutpred. mutdb.org/) (27), and MutationTaster (http://www. mutationtaster.org/) (28). We also applied VarSome, an integrated search engine that allows accessing multiple pathogenicity prediction tools and databases (https:// varsome.com/) (29). In this case, variant pathogenicity is reported using an automatic classifier that evaluates the submitted variant according to the American College of Medical Genetics and Genomics (ACMG) guidelines (30). Protein stability modifications resulting from missense variants were assessed with web-based programs MUpro (http://mupro.proteomics.ics.uci. edu/) (31) and INPS (https://inpsmd.biocomp.unibo. it/inpsSuite) (32) using the OCRL1 protein sequence. These applications provide the calculated free energy change value (DDG). A DDG value below zero indicates that the stability of the protein has decreased, whereas a DDG higher than zero means it has increased. The 
protein sequence of human OCRL1 (901 amino acids) was obtained from the NCBI database (https://www.ncbi. nlm.nih.gov/protein/; accession number NP_000267.2).

\subsection{Protein modeling}

The three-dimensional (3-D) structures of wild-type and mutant ASH domains were predicted using the online modeling server SWISS-MODEL (https://swissmodel. expasy.org/) with the crystal structure of OCRL1 (amino acid residues 540-678) in complex with Rab8a:GppNHp (3qbt.3.B) as a template (21).

\section{Results}

\subsection{Clinical characteristics of patients}

Patient 1, a 16-year-old boy from Spain, was the third child of a healthy mother. His father had hypercholesterolemia. The patient's medical history revealed that he was born at term after a normal pregnancy. His birth weight was $3,200 \mathrm{~g}$. Ophthalmological examination at the age of 2 months revealed bilateral congenital cataracts, and he had cataract surgery at three months of life. He showed mild muscular hypotonia and mild psychomotor retardation, but the results of neuroimaging tests were normal. In the first years of life he also presented ocular nystagmus with horizontal wide-angle eye movements, a slight coordination impairment in rapid alternating movements of upper limbs, a distinctive facial expression, and initial rejection of solid foods due to difficulty in chewing that has improved with speech therapy treatment. Lowe syndrome was suspected, and a renal function study was performed that confirmed data of proximal tubulopathy with incomplete Fanconi syndrome (hypercalciuria and tubular proteinuria). Glycosuria, phosphaturia and metabolic acidosis were not observed. His clinical evolution was appropriate, with a normal estimated glomerular filtration rate $\left(103 \mathrm{ml} / \mathrm{min} / 1,73 \mathrm{~m}^{2}\right)$. He maintained a moderate hypercalciuria (calcium/creatinine ratio of $0.3-0.4 \mathrm{mg} /$ $\mathrm{mg}$ ) until 10 years of age when it was normalized. The urinary elimination of citrate was adequate (citrate/ creatinine ratios $>300 \mathrm{mg} / \mathrm{g}$ ), with some hypocitraturia in periods of higher calciuria. Ultrasound examination showed that he had not developed nephrocalcinosis. His tubular proteinuria is moderate, with protein/ creatinine ratios between $1-0.8 \mathrm{mg} / \mathrm{mg}$ at the expense of $\beta_{2}$ microglobulin. In the psychometric assessment at age 14 , administration of the Wechsler Intelligence Scale for Children-IV, showed that his intelligence quotient was 67 . The child is in school with curricular adaptation and psychoeducational support.

Patient 2 was an 11-year-old boy from Brazil whose mother descends from a Spanish family. He was born with 37 weeks gestation. His birth weight and length were $3,120 \mathrm{~g}$ and $50 \mathrm{~cm}$, respectively. During pregnancy his mother had hypertension and polyhydramnios. His sister was healthy. Muscular hypotonia and congenital cataracts were diagnosed at the age of 1 month. Clinical tests showed proteinuria by the age of 9 months. He evolved with delayed development (cognitive deficit) and partial loss of vision due to bilateral congenital cataracts that were corrected surgically, glaucoma and nystagmus. The child presents typical facies of Lowe syndrome with front protrusion and deep eyes, and short stature (-2.3 SD). He also has attention deficit disorder and hyperactivity. Further examination revealed metabolic acidosis, hypercalciuria, hyperuricosuria and proteinuria (protein/creatinine ratios between 8.7-4.4 mg/mg), and no nephrocalcinosis. His last estimated glomerular filtration rate is normal $(111 \mathrm{ml} /$ $\left.\min / 1,73 \mathrm{~m}^{2}\right)$.

3.2. Identification of novel pathogenic $O C R L$ variants and bioinformatics predictions

To confirm the clinical diagnosis of Lowe syndrome, we carried out a mutational analysis of the $O C R L$ in the two patients and his relatives. The results revealed two novel hemizygous variants in exon 18 (Figure 1). These results were confirmed by bidirectional sequencing of independent PCR products. The variant identified in patient 1 consisted in the substitution of a $\mathrm{T}$ for an A in codon 636 , which predicts the change of valine for glutamic acid in the OCRL1 protein (c.1907T $>$ A; p.V636E; g.36496T $>$ A; Chr23:128710321 T $>$ A; Ensembl transcript ID: ENST00000371113) (Figure 1A). His mother and one of his sisters were healthy carriers of this mutation, while the father and the other sister showed the normal sequence (Figure 1A). The variant detected in patient 2 involved an $A$ to $C$ change in codon 660 that predicts the substitution of histidine for proline (c.1979A $>$ C; p.H660P; g.36568A $>$ C; Chr23:128710393A $>$ C; Ensembl transcript ID: ENST00000371113) (Figure 1B). The mother of patient 2 was not a carrier of the variant, suggesting this mutation occurred de novo (Figure 1B). His father also displayed the normal sequence. Examination of the protein sequence indicates that both variants are located in the ASH domain of OCRL1 (Figure 2). The amino acid residues affected by these two variants are highly conserved among different species (human, mouse, pig, chicken, fish and frog) (Figure 2B), and, therefore, they are probably important for the structure and function of the protein. These $O C R L$ variants, c.1907T >A (p.V636E) and c.1979A >C (p.H660P), had not been reported in the literature and were absent from population and clinical databases including gnomAD, ClinVar, 1000 Genomes Project, and Human Gene Mutation Database. Interestingly, the search in gnomAD retrieved variant p.V636M, found in only one allele (frequency in total population 5.46e-6), which has not been associated with disease. The novel variants 
A

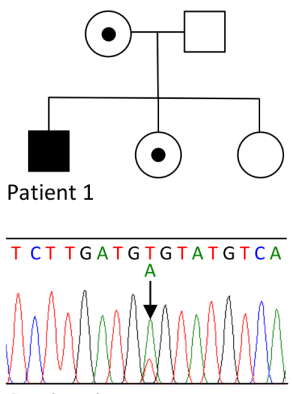

Carrier sister

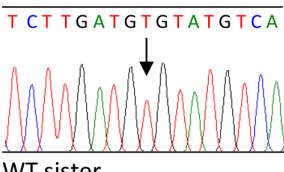

B
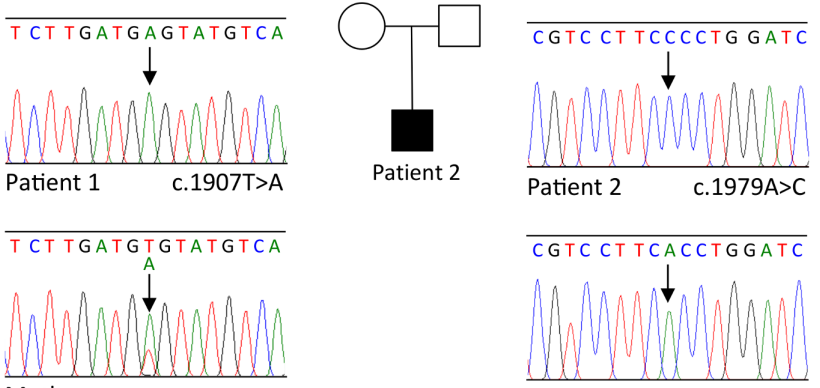

Mother

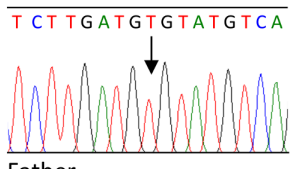

Father

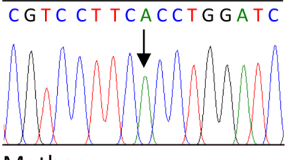

Mother

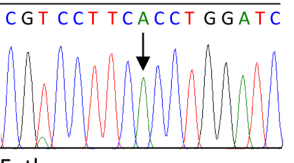

Father

Figure 1. Novel $O C R L$ mutations identified in two patients with Lowe syndrome. (A) Partial DNA sequence of $O C R L$ exon 18 in patient 1 and his relatives, and family pedigree. Arrows indicate the altered nucleotide position and hemizygous mutation c.1907T >A, p.V636E in the patient. The patient's mother and one of his sisters are carriers of the mutation. The father and the other sister (WT, wild-type) show the normal sequence. (B) Partial sequence of exon 18 of $O C R L$ in patient 2 and his parents, and family pedigree. Arrows indicate the affected nucleotide position and hemizygous mutation c.1979A $>$ C, p.H660P in the patient. Both parents show the normal sequence suggesting the mutation was originated de novo. Circles and squares represent female and male individuals, respectively; black squares represent affected individuals; black circles within open frames denote heterozygous individuals; open circles and squares represent family members with the normal sequence.

A

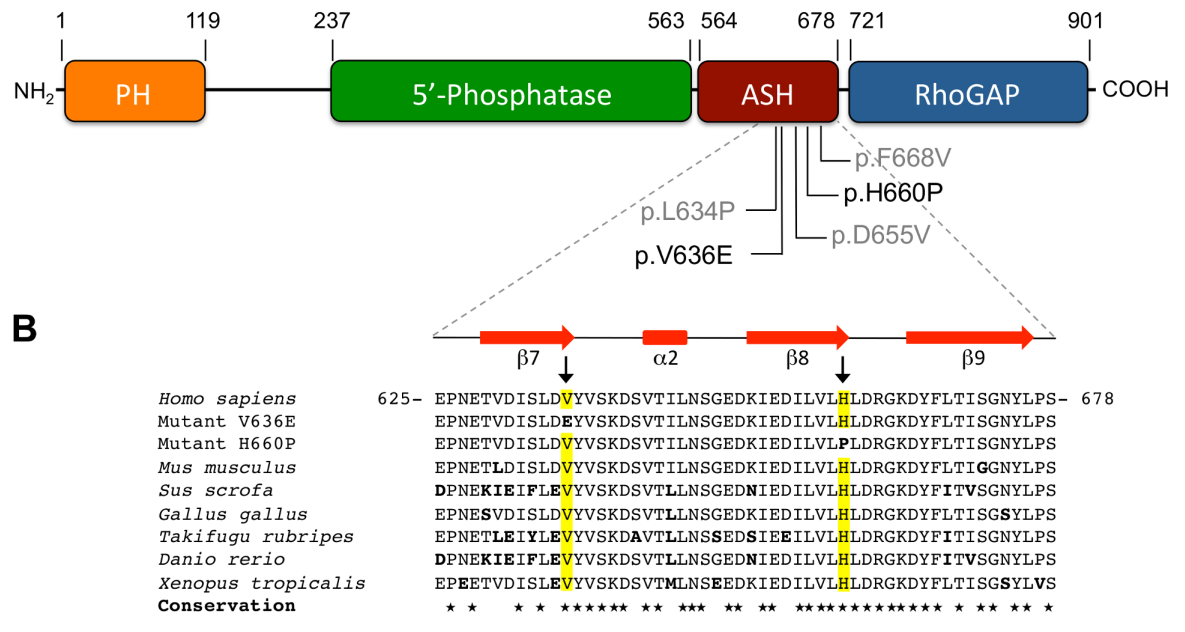

Figure 2. Schematic representation of OCRL1 and location of mutations in the ASH domain. (A) The different protein domains are shown: Pleckstrin homology domain (PH), 5-phosphatase catalytic domain, ASPM, SPD-2, Hydin domain (ASH) and RhoGAP-like domain. The two missense mutations identified in this study, p.V636E and p.H660P, and three previously reported mutations, p.L634P, p.D655V and p.F668V $(3,20,23)$ are indicated in black and grey, respectively. (B) Multiple sequence alignment analysis of the ASH domain showing evolutionary conservation of valine 636 and histidine 660 (yellow background) among OCRL1 proteins. These amino acid residues are located in $\beta$-sheets 7 ( $\beta 7$ ) and 8 ( $\beta 8)$, respectively. Regular and bold letters represent fully conserved and non-conserved residues, respectively.

described here were submitted to ClinVar, National Center for Biotechnology Information (https://www.ncbi. nlm.nih.gov/clinvar) and were included with accession numbers VCV000689472.1 and VCV000689471.1 for c.1907T $>$ A (p.V636E) and c.1979A $>$ C (p.H660P), respectively.

Bioinformatics analysis of p.V636E with five different bioinformatics tools predicted that this variant is pathogenic (Table 1). In addition, the software VarSome classified this substitution as "likely pathogenic" because it met two moderate (PM1 and PM2) and three supporting (PP2, PP3 and PP5) pathogenicity criteria established by the ACMG. All thirteen algorithms included in VarSome inferred the conclusion of pathogenicity. Furthermore, variant p.V636E caused a decrease in OCRL1 protein stability according to programs that predict protein stability of mutated proteins including INPS (DDG in kcal/mol: -2.447) and 
Table 1. Bioinformatics predictions of pathogenicity for $O C R L$ mutations affecting the ASH domain

\begin{tabular}{|c|c|c|c|c|c|}
\hline Mutation & $\begin{array}{l}\text { SIFT } \\
\text { (score) }\end{array}$ & $\begin{array}{l}\text { PolyPhen-2 } \\
\text { (score) }\end{array}$ & $\begin{array}{l}\text { CADD } \\
\text { (score) }\end{array}$ & $\begin{array}{l}\text { MutationTaster } \\
\text { (score) }\end{array}$ & $\begin{array}{l}\text { MutPred2 } \\
\text { (score) }\end{array}$ \\
\hline p.V636E & $\begin{array}{l}\text { Affects function } \\
\qquad(0.00)\end{array}$ & $\begin{array}{l}\text { Probably damaging } \\
\qquad(0.999)\end{array}$ & $\begin{array}{l}\text { Damaging } \\
(27.9)\end{array}$ & $\begin{array}{l}\text { Disease causing } \\
\qquad(121)\end{array}$ & $\begin{array}{l}\text { Pathogenic } \\
(0.858)\end{array}$ \\
\hline p.H660P & $\begin{array}{l}\text { Affects function } \\
\qquad(0.00)\end{array}$ & $\begin{array}{c}\text { Probably damaging } \\
(0.998)\end{array}$ & $\begin{array}{l}\text { Damaging } \\
(26.6)\end{array}$ & $\begin{array}{c}\text { Disease causing } \\
\text { (77) }\end{array}$ & $\begin{array}{c}\text { Pathogenic } \\
(0.915)\end{array}$ \\
\hline
\end{tabular}

Amino acid substitutions with SIFT scores $<0.05$ are predicted to be deleterious (scores closer to 0 are more confidently predicted to be deleterious). Variants with HumVar PolyPhen-2 scores in the range 0.85 to 1.0 are more confidently predicted to be damaging. CADD-PHRED scaled CADD score equal or higher than 15 indicates that the variant is damaging. The MutationTaster score is taken from the Grantham Matrix for amino acid substitutions and it ranges from 0.0 to 215 . The general score of MutPred 2 ranges from 0.0 and 1.0, with a higher score indicating a greater predisposition to be pathogenic.

A

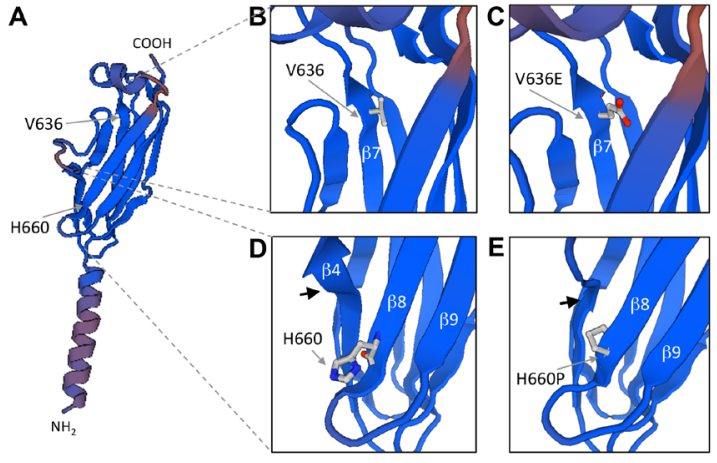

Figure 3. Protein structure modeling of wild-type and mutated ASH domains in ribbon-presentation. The figure shows the ASH domain and $\alpha$-helix 1 of the 5-phosphatase domain (amino acid residues 564-678). The ASH domain of OCRL1 (residues 564-678) is composed of nine $\beta$-strands and a small $\alpha$-helix. The $\beta$-sheets form two layers. Hydrophobic side chains from the two layers occupy the interior of the sandwich. (B and C) Close-ups showing wildtype valine 636 and mutant glutamic acid 636, respectively. (D and E) Close-ups showing wild-type histidine 660 and mutant proline 660 , respectively. The black arrow indicates the $\beta$-strand 4 ( $\beta 4$ ) that appears shifted in the mutants ASH domain.

MuPro (DDG in $\mathrm{kcal} / \mathrm{mol}:-1.632)$. In silico analysis of the amino acid change resulting from p.H660P showed that this variant also has a deleterious effect on OCRL1 protein function (Table 1). VarSome analysis classified this variant as likely pathogenic because it met two moderate (PM1 and PM2) and three supporting (PP2, PP3 and PP5) pathogenicity criteria established by the ACMG (30). The VarSome pathogenic outcome derived from predictions from eleven out of fourteen scores (versus 3 benign predictions). Furthermore, variant p.H660P caused a decrease in OCRL1 protein stability according to both INPS (DDG in kcal/mol: -1.022) and MuPro (DDG in kcal/mol: -0.924) programs.

\subsection{The 3-D structure of mutant ASH domains}

To evaluate the structural impact of missense mutations p.V636E and p.H660P, we constructed 3-D models of the wild-type and mutants ASH domains using the SWISS-MODEL software (Figure 3). The protein sequence used contained, in addition to the ASH domain, $\alpha$-helix 1 of the 5-phosphatase domain (amino acid residues 540-678). Amino acid residue V636 is at the $\mathrm{C}$-terminal end of $\beta$-strand 7 that is buried in the core of the ASH domain (Figure 2 and Figure 3). The mutant residue in $\mathrm{p} . \mathrm{V} 636 \mathrm{E}$ has a larger and less hydrophobic side chain than the wild-type residue, which would cause loss of hydrophobic interactions in the ASH domain core. Additionally, the charge of the mutant residue is negative while the wild-type residue charge is neutral. These differences in amino acid properties could lead to disruptions in proper folding and function. Conversely, residue H660 is positioned close to the $\mathrm{C}$-terminal end of $\beta$-strand 8 , which is on the surface of the ASH domain (Figure 2 and Figure 3). The mutant residue, proline, is smaller and more hydrophobic than the wild-type residue. The size difference between wild-type and mutant residues makes that the new residue is not in the correct position to create the same hydrogen bond as the original residue did. In addition, the difference in hydrophobicity will affect hydrogen bond formation causing a likely loss of external interactions. Also, the predicted 3-D of the mutant p.H660P ASH domain shows a displacement of $\beta$-strand 4 (Figure 3E).

\section{Discussion}

Patients with Lowe syndrome usually present in childhood with cataracts, progressive growth failure, hypotonia, and proximal tubulopathy, although a wide clinical variability among patients has been described (1). The clinical diagnosis can be confirmed by mutational analysis of the $O C R L$ gene. More than two hundred $O C R L$ variants associated with Lowe syndrome have been identified. Most of the missense variants modify conserved residues in the 5-phosphatase domain, altering its folding, substrate specificity or catalytic activity $(2,14)$. There are also several pathogenic missense mutations located in the ASH and RhoGAP domains, some of which abolish interactions with OCRL1 partners and alter the cellular localization of the OCRL1 protein (19-22). Fibroblasts derived from Lowe syndrome patients harboring some 
of these mutations have reduced 5-phosphatase activity, suggesting that mutations in the ASH and RhoGAP domains somehow disturb OCRL1 activity $(17,33)$. These two domains are widely interconnected and form a single folding module; therefore, the destabilization of one of them will affect the stability of the other (12). The ASH-RhoGAP module mediates the interactions of OCRL1 with protein partners that facilitate its targeting to different cellular locations such as early endosomes, Golgi complex, lysosomes and primary cilium $(2,19)$. The ASH domain has a $\beta$-sheet structure similar to the immunoglobulin $\mathrm{G}$ domain and contains a Rabbinding site that allows the interaction of OCRL1 with Rab GTPases $(12,21,34)$. This interaction is needed for targeting of OCRL1 to the Golgi complex and endosomal membranes. Mutations in the Rab-binding site perturb the interaction between the OCRL1 protein and its partners, and result in membrane targeting defects. In the present study, we analyzed by DNA sequencing the $O C R L$ gene of two unrelated patients with the clinical diagnosis of Lowe syndrome; both presented congenital cataracts, developmental delay (mild in one of them), muscular hypotonia and proximal tubulopathy. Two novel missense variants, c.1907T $>$ A (p.V636E) and c.1979A $>$ C (p.H660P) were identified, and both are located in the ASH domain. Variant c.1979A $>$ C (p.H660P) seems to have originated from the patient's DNA or from his mother's germ cells. Genetic studies of mothers of affected males have shown the existence of de novo mutation in 30 to $37 \%$ of cases $(17,18)$.

The ASH domain is comprised of two layers of $\beta$-sheets forming a sandwich, and hydrophobic side chains from the two layers occupy the interior space. Amino acid residue valine 636 is located in $\beta$-strand 7 of the ASH domain and its replacement for glutamic acid causes loss of hydrophobic interactions in the interior of the ASH domain. Therefore, we suggest that mutation p.V636E causes a folding defect in the ASH domain. Another Lowe syndrome-causing mutation, p.L634P, located very close to valine 636 on the same $\beta$-strand 7 , seems also to disrupt the correct structure of the ASH domain and the ASH-RhoGAP module, and has been shown to abolish binding to the endocytic protein APPL1 $(20,22)$. The histidine residue affected by mutation p.H660P is located on $\beta$-strand 8 on the surface of the ASH domain. The substitution of histidine for proline could impair binding of OCRL1 with other proteins, and, therefore, disturb its function. Furthermore, histidine 660 forms a hydrogen bond with the aspartic acid in position 666 on $\beta$-strand 9, which is involved in the interaction of the ASH domain with Rab8a (21). Therefore, mutation p.H660P could affect this interaction and OCRL1 membrane recruitment. Two known pathogenic mutations p.D655V and p.F668V, located in $\beta$-strands 8 and 9 , respectively, on the same face of the ASH domain have been shown to abolish binding of OCRL1 to Rab proteins (21-23). We should also take into account that some missense mutations located in the ASH-RhoGAP module can destabilize OCRL1 (17), and in fact, both mutations describe in our study reduce the protein stability according to bioinformatics predictions.

In conclusion, the identification of two novel missense mutations located in the ASH domain may shed more light on the functional importance of this domain. Our results extend the range of $O C R L$ pathogenic mutations in patients with Lowe syndrome. Based on the results obtained with other patient mutations located in the ASH-RhoGAP-like domain of OCRL1, we suggest that p.V636E and p.H660P cause Lowe syndrome by disrupting the interaction between OCRL1 and some of its partners or by destabilizing de protein.

Funding: This work is part of the RenalTube project and was supported by grant PI17/00153 integrated in the Plan Nacional de I+D+I 2013-2016 and co-financed by the ISCIII-Subdirección General de Evaluación y Fomento de la Investigación and the European Regional Development Fund "Another way to build Europe". We are grateful to the families for their participation in this study.

Conflict of Interest: The authors have no conflict of interest to disclose.

\section{References}

1. Bökenkamp A, Ludwig M. The oculocerebrorenal syndrome of Lowe: an update. Pediatr Nephrol. 2016; 31:2201-2212.

2. De Matteis MA, Staiano L, Emma F, Devuyst O. The 5-phosphatase OCRL in Lowe syndrome and Dent disease 2. Nat Rev Nephrol. 2017; 13:455-470.

3. Bockenhauer D, Bokenkamp A, van't Hoff W, Levtchenko E, Kist-van Holthe JE, Tasic V, Ludwig M. Renal phenotype in Lowe syndrome: a selective proximal tubular dysfunction. Clin J Am Soc Nephrol. 2008; 3:1430-1436.

4. Attree O, Olivos IM, Okabe I, Bailey LC, Nelson DL, Lewis RA, McInnes RR, Nussbaum RL. The Lowe's oculocerebrorenal syndrome gene encodes a protein highly homologous to inositol polyphosphate-5phosphatase. Nature. 1992; 358:239-242.

5. Olivos-Glander IM, Jänne PA, Nussbaum RL. The oculocerebrorenal syndrome gene product is a 105$\mathrm{kD}$ protein localized to the Golgi complex. Am J Hum Genet. 1995; 57:817-823.

6. Nussbaum RL, Jänne PA, Charnas L, Chinault AC, Orrison BM. Physical mapping and genomic structure of the Lowe syndrome gene OCRL1. Hum Genet. 1997; 99:145-150.

7. Cau M, Addis M, Congiu R, Meloni C, Cao A, Santaniello S, Loi M, Emma F, Zuffardi O, Ciccone R, Sole G, Melis MA. A locus for familial skewed X chromosome inactivation maps to chromosome Xq25 in a family with a female manifesting Lowe syndrome. J Hum Genet. 2006; 51:1030-1036.

8. Recker F, Reutter H, Ludwig M. Lowe syndrome/Dent2 disease: a comprehensive review of known and novel 
aspects. J Pediatr Genet. 2015; 2:53-68.

9. Hoopes RR Jr, Shrimpton AE, Knohl SJ, Hueber P, Hoppe B, Matyus J, Smickes A, Tasic V, Toenshoff B, Suchy SF, Nussbaum RL, Scheinman SJ. Dent disease with mutations in OCRL1. Am J Hum Genet. 2005; 76:260-267.

10. Bökenkamp A, Böckenhauer D, Cheong HI, Hoppe B, Tasic V, Unwin R, Ludwig M. Dent-2 disease: a mild variant of Lowe syndrome. J Pediatr. 2009; 155:94-99.

11. Claverie-Martín F, Ramos-Trujillo E, García-Nieto V. Dent's disease: clinical features and molecular basis. Pediatr Nephrol. 2010; 26:693-704.

12. Erdmann KS, Mao Y, McCrea HJ, Zoncu R, Lee S, Paradise S, Modregger J, Bimesderfer D, Toomre D, De Camilli P. A role of the Lowe syndrome protein OCRL in early steps of the endocytic pathway. Dev Cell. 2007; 13:377-390.

13. Mao Y, Balkin DM, Zoncu R, Erdmann KS, Tomasini L, Hu F, Jin MM, Hodsdon ME, De Camilli P. A PH domain within OCRL bridges clathrin-mediated membrane trafficking to phosphoinositide metabolism. EMBO J. 2009; 28:1831-1842.

14. Pirruccello M, De Camilli P. Inositol 5-phosphatases: insights from the Lowe syndrome protein OCRL. Trends Biochem Sci. 2012; 37:134-143.

15. Loi M. Lowe syndrome. Orphanet J Rare Dis. 2006; 1:16.

16. Monnier N, Satre V, Lerouge E, Berthoin F, Lunardi J. OCRL1 mutation analysis in French Lowe syndrome patients: implications for molecular diagnosis strategy and genetic counseling. Hum Mutat. 2000; 16:157-165.

17. Hichri H, Rendu J, Monnier N, Coutton C, Dorseuil O, Vargas-Poussou R, Baujat G, Blanchard A, Nobili F, Ranchin B, Remesy M, Salomon R, Satre V, Lunardi J. From Lowe syndrome to Dent disease: correlations between mutations of the OCRL1 gene and clinical and biochemical phenotypes. Hum Mutat. 2011; 32:379-388.

18. Recker F, Zaniew M, Böckenhauer D, et al. Characterization of 28 novel patients expands the mutational and phenotypic spectrum of Lowe syndrome. Pediatr Nephrol. 2015; 30:931-943.

19. McCrea HJ, Paradise S, Tomasini L, Addis M, Melis MA, De Matteis MA, De Camilli P. All known patient mutations in the ASH-RhoGAP domains of OCRL affect targeting and APPL1 binding. Biochem Biophys Res Commun. 2008; 369:493-499.

20. Swan LE, Tomasini L, Pirruccello M, Lunardi J, De Camilli P. Two closely related endocytic proteins that share a common OCRL-binding motif with APPL1. Proc Natl Acad Sci USA. 2010; 107:3511-3516.

21. Hou X, Hagemann N, Schoebel S, Blankenfeldt W, Goody RS, Erdmann KS, Itzen A. A structural basis for Lowe syndrome caused by mutations in the Rab-binding domain of OCRL1. EMBO J. 2011; 30:1659-1670.

22. Pirruccello M, Swan LE, Folta-Stogniew E, De Camilli P. Recognition of the F\&H motif by the Lowe syndrome protein OCRL. Nat Struct Mol Biol. 2011; 18:789-795.

23. Shalaby AK, Emery-Billcliff P, Baralle D, Dabir T, Begum S, Waller S, Tabernero L, Lowe M, Self J. Identification and functional analysis of a novel oculocerebrorenal syndrome of Lowe $(O C R L)$ gene variant in two pedigrees with varying phenotypes including isolated congenital cataract. Mol Vis. 2018; 24:847-852.

24. Sim NL, Kumar P, Hu J, Henikoff S, Schneider G, Ng PC. SIFT web server: predicting effects of amino acid substitutions on proteins. Nucleic Acids Res. 2012; 40:W452-W457.

25. Adzhubei IA, Schmidt S, Peshkin L, Ramensky VE, Gerasimova A, Bork P, Kondrashov AS, Sunyaev SR. A method and server for predicting damaging missense mutations. Nat Methods. 2010; 7:248-249.

26. Rentzsch P, Witten D, Cooper GM, Shendure J, Kircher M. CADD: predicting the deleteriousness of variants throughout the human genome. Nucleic Acids Res. 2018; 47:D886-D894.

27. Pejaver V, Mooney SD, Radivojac P. Missense variant pathogenicity predictors generalize well across a range of function-specific prediction challenges. Hum Mutat. 2017; 38:1092-1108.

28. Schwarz JM, Cooper DN, Schuelke M, Seelow D. MutationTaster2: mutation prediction for the deepsequencing age. Nat Methods. 2014; 11:361-362.

29. Kopanos C, Tsiolkas V, Kouris A, Chapple CE, Albarca Aguilera M, Meyer R, Massouras A. VarSome: the human genomic variant search engine. Bioinformatics. 2018; 35:1978-1980.

30. Richards S, Aziz N, Bale S, Bick D, Das S, GastierFoster J, Grody WW, Hegde M, Lyon E, Spector E, Voelkerding K, Rehm HL; ACMG Laboratory Quality Assurance Committee. Standards and guidelines for the interpretation of sequence variants: a joint consensus recommendation of the American College of Medical Genetics and Genomics and the Association for Molecular Pathology. Genet Med. 2015; 17:405-424.

31. Cheng J, Randall A, Baldi P. Prediction of protein stability changes for single-site mutations using support vector machines. Proteins. 2005; 62:1125-1132.

32. Savojardo C, Fariselli P, Martelli PL, Casadio R. INPSMD: a web server to predict stability of protein variants from sequence and structure. Bioinformatics. 2016; 32:2542-2544.

33. Kawano T, Indo Y, Nakazato H, Shimadzu M, Matsuda I. Oculocerebrorenal syndrome of Lowe: three mutations in the OCRL1 gene derived from three patients with different phenotypes. Am J Med Genet. 1998; 77:348-355.

34. Hyvola N, Diao A, McKenzie E, Skippen A, Cockcroft $\mathrm{S}$, Lowe M. Membrane targeting and activation of the Lowe syndrome protein OCRL1 by rab GTPases. EMBO J. 2006; 25:3750-3761.

Received August 20, 2020; Revised October 6, 2020; Accepted October 9, 2020.

\section{*Address correspondence to:}

Félix Claverie-Martín, Unidad de Investigación, Hospital Nuestra Señora de Candelaria, Carretera del Rosario 145, 38010 Santa Cruz de Tenerife, Spain.

E-mail: fclamar@gobiernodecanarias.org

Released online in J-STAGE as advance publication October $12,2020$. 